\title{
DEVELOPMENT OF A TWO-WHEEL CONTINGENCY MODE FOR THE MAP SPACECRAFT
}

\author{
Scott R. Starin and James R. O'Donnell, Jr., Ph.D. \\ NAS.4/Goddard Space Flight Center, Greenbelt, MD 20771
}

\begin{abstract}
$\underline{\text { Abstract }}$
The Microwave Anisotropy Probe (MAP) is a followon mission to the Cosmic Background Explorer $(\mathrm{COBE})$, and is currently collecting data from its orbit near the second Sun-Earth libration point. Due to limited mass, power, and financial resources, a traditional reliability concept including fully redundant components was not feasible for MAP. Instead, the MAP design employs selective hardware redundancy in tandem with contingency software modes and algorithms to improve the odds of mission success. One direction for such improvement has been the development of a two-wheel backup control strategy. This strategy would allow MAP to position itself for maneuvers and collect science data should one of its three reaction wheels fail. Along with operational considerations, the strategy includes three new control algorithms. These algorithms would use the remaining attitude control actuators-thrusters and two reaction wheels-in ways that achieve control goals while minimizing adverse impacts on the functionality of other subsystems and software.
\end{abstract}

\section{Introduction}

The MAP Observatory nominally uses three reaction wheels for most of its attitude control requirements. See Andrews et $a l^{1}$ and O'Donnell et $a l^{2}$ for further information on the MAP Attitude Control System (ACS). In the event of a failure of one of the three reaction wheel assemblies (RWAs), it would not be possible to achieve full, three-axis control using the remaining two wheels. Consequently, two of the attitude control algorithms implemented on the MAP spacecraft would no longer be usable. The two most affected modes would be Inertial Mode, which slews to and holds inertial attitudes, and science Mode, which implements the nominal dual-spin science mode.

During early design of the MAP Observatory, a careful cost-benefit analysis resulted in the omission of a redundant fourth wheel from the ACS design; this decision was reached in part because a Safehold mode using only two-wheels was feasible. At a Red

Copyright $(C) 2001$ by the American Institute of Aeronautics and Astronautics, Inc. No copyright is asserted in the United States under Title 17, U.S. Code. The U.S. Government has a royalty-free license to exercise all rights under the copyright claimed herein for Governmental Purposes. All other rights are reserved by the copyright owner.
Team Review (Sept. 12-13, 2000), as a result of discussion on the possibility of software-based RWA redundancy over the entire mission, the pre-launch development of a strategy for completing the mission with two operable RWAs became an imperative. This paper will discuss the three principal algorithms designed to achieve software-based RWA redundancy: Thruster-Based Inertial Mode, TwoWheel Science Mode, and Momentum Adjust. In addition, there is a description of the other changes that would need to be made to the MAP onboard flight software for it to fulfill a science mission degraded by the loss of a wheel.

\section{Wheel Failure Contingencv Design}

In order to deliver and implement a backup twowheel control design in a timely fashion, a design philosophy was first adopted. ${ }^{2}$ When possible, existing control algorithms were used with few or no changes to reduce development and testing time. Where new algorithms were needed, they were implemented in a manner consistent with the current flight software design, using modifiable tables to allow for on-orbit flexibility. Finally, the development of new and changed algorithms was prioritized by when they were needed to maximize the chances of mission success in the event of a wheel failure at any point in the mission. This last tenet also served to maintain proper focus on other spacecraft concerns as the actual launch approached.

\section{Mission Functions and Implementation Plan}

Several ACS functions were required to carry out the MAP mission. The following describes how the various functions would be affected by the loss of a wheel, and what design steps were necessary to prepare for the possibility of two-wheel operations.

Safehold: In the event of a wheel failure, the Safehold control mode is capable of directing the spacecraft $Z$-axis, and therefore the solar arrays, toward the Sun and holding an instrument-safe, power-positive condition until new software can be uploaded. The existing MAP Safehold/CSS (CSS: coarse Sun sensor) controller can work with only two wheels if the system momentum bias is small. The two-wheel Safehold/CSS also works with a large momentum bias as long as the bias is close to the Sun vector. 
Thruster Maneuvers: To fulfill its mission, MAP had to be able to perform a number of orbit maneuvers during phasing loops about the Earth to get to $\mathrm{L}_{2}$. Now at $L_{2}$, occasional stationkeeping maneuvers must also be perfurmed. The existing MAP Delta $\mathrm{V}$ Mode has been performing this function in actuality, and could have performed for the two-wheel case as well, had it been necessary. However, with the loss of the wheel-based Inertial Mode, an alternative way would have been needed to get the spacecraft into the correct attitude for these thruster operations. With two relatively small changes to the existing Delta $V$ controller, it would be capable of this function, becoming a Thruster-Based Inertial Mode.

Science Observations: The Two-Wheel Science Mode uses a momentum bias about the Sun vector; its core algorithm will be discussed below. A big difference between the nominal and Two-Wheel science modes is that the nominal mode performs its dual spin about the Sun vector, while the two-wheel mode would perform its dual spin about a momentum bias, nominally applied about the Sun vector. The existing MAP Delta $\mathrm{H}$ Mode can be used to establish or remove the science mode momentum bias using thrusters. This momentum bias is fixed in inertial space, however, and will move relative to the Sun vector approximately $1 \%$ day. A new thruster control mode is needed to perform the small, daily adjustments to the momentum bias that will be necessary in flight. This Momentum Adjust Mode will also be discussed in a later section.

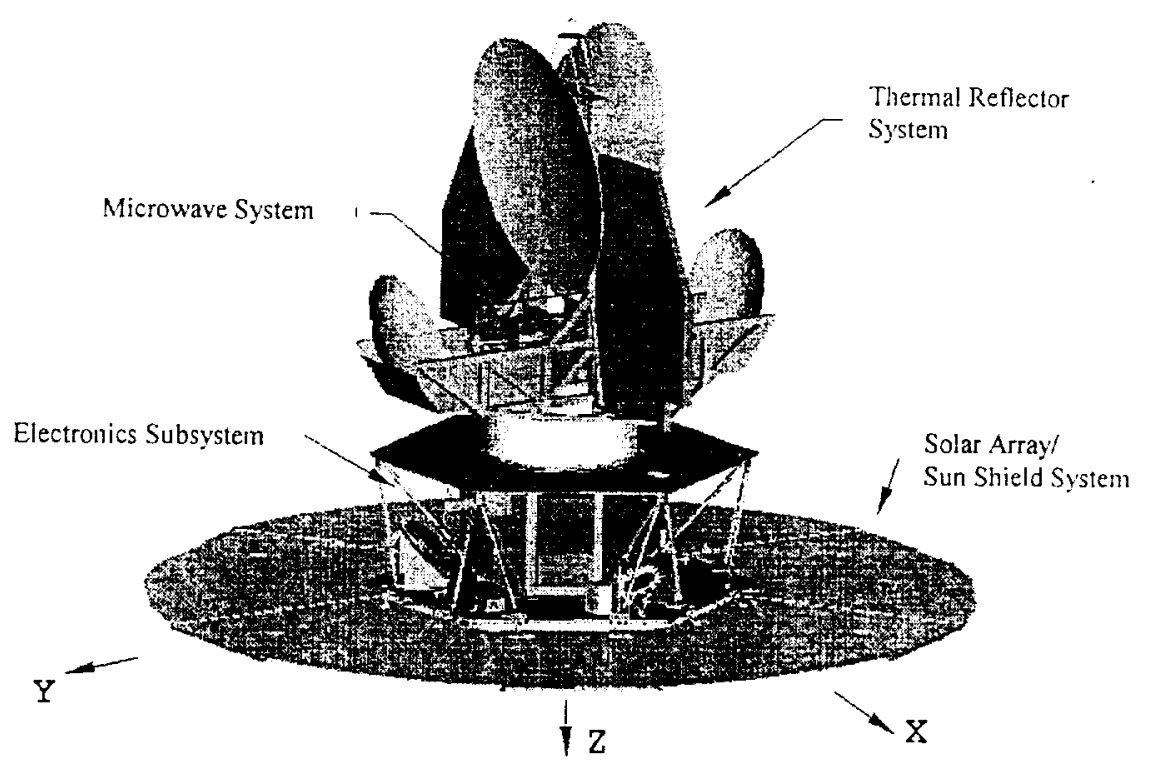

Figure 1: Configuration of MAP spacecraft with solar arrays deployed

\section{New Algorithms}

Development of two-wheel flight software was completed in two phases. In the pre-launcli phase, a software patch to install the Thruster-Based Inertial Mode was developed and tested, as this was the only new algorithm needed to get MAP to $\mathrm{L}_{2}$. In the second phase, the other two algorithms needed for MAP to fulfill the remainder of its science mission in the event of a wheel failure were developed and tested. These are called Two-Wheel Science and Momentum Adjust.

\section{Thruster-Based Inertial Mode}

The current Delta $V$ Mode is meant for thruster operations, not simply holding an attitude. It automatically exits when the commanded velocity change $(\Delta V)$ equals the $\Delta V$ inparted during the burn. This means that if a new attitude is commanded with $\Delta \mathrm{V}=0$, the mode immediately interprets the $\Delta \mathrm{V}$ as complete and exits without firing the thrusters at all. For the mode to be used as a Thruster-Based Inertial Mode independent of an orbit maneuver, an option to allow for a zero- $\Delta \mathrm{V}$ command to the Delta $\mathrm{V}$ Mode was necessary. If commanded in this fashion, the 
mode would only exit when it timed out, with the time limit defined by a flight software table value, or upon command into another control mode, including a conventional Delta $V$ maneuver.

The existing Delta $V$ Mode was not meant to execute large inertial slews; attempting to do so would result in gyro rate saturation because the thruster torque authority is large and the algorithm does not include any rate-limiting action. In the two-wheel contingency design, an attitude limiter would be added to the Delta V Mode controller, effectively limiting its slew rate and allowing it to be used for slews of arbitrary magnitude.

Once designed, the Thruster-Based Inertial Mode was included in the pre-launch software patch; that patch was to be uploaded to MAP if a wheel failed any time between launch and the development of the full contingency science operations patch. This patch was much simpler than the full, envisioned patch since it did not need to include science operations, and it was designed with a rapid upload in mind. This preliminary patch would have greatly improved chances for the spacecraft to complete its phasing loops and lunar gravity assist had a wheel failed during the critical maneuvering phase of the mission (the first six weeks).

\section{Two-Wheel Science Mode}

The design of the Two-Wheel Science Mode has been described in a previous paper; the following discussion details the portions that contributes to an understanding of the two-wheel contingency design as a whole.

MAP Configuration: The MAP Observatory is equipped with three reaction wheels, which are arranged symmetrically about the spacecraft $Z$-axis. Reaction Wheel Assembly 1 (RWAl) lies in the $+X /-Z$ quarter-plane of the spacecraft body reference frame, and had a $30^{\circ}$ tilt with respect to the $X Y$ plane; RWA2 and RWA3 have the same $30^{\circ}$ tilt. The nominal-mission science mode uses the three reaction wheels to establish commanded Euler angle rates. ${ }^{3} \mathrm{~A}$ 3-1-3 Euler angle rotation is used, with the angles named $\phi, \theta$ and $\psi$, respectively. The rates to be commanded are:

$$
\begin{aligned}
& \dot{\varphi}=1 \mathrm{rph}=0.1^{\circ} / \mathrm{sec} \\
& \dot{\theta}=0^{\circ} / \mathrm{sec} ; \text { with } \theta=22.5^{\circ} \\
& \dot{\psi}=0.464 \mathrm{rpm}=2.8^{\circ} / \mathrm{sec}
\end{aligned}
$$

where rph is revolutions per hour, and rpm is revolutions per minute) The angle $\theta$, which is the angle between the Sun vector and the spacecraft $Z$ axis, is maintained at a constant value of $22.5^{\circ}$. The rate in $\psi$ represents a spin about the $Z$-axis, and the rate in $\phi$ is therefore a precession of the $Z$-axis in inertial space.

The Whet Failure Problem: The flight software establishes the commanded rates by continually generating a small quaternion adjustment, $\Delta q$, and then commanding the wheels to enact that $\Delta q$. Because the system momentum is small, tiny corrections in the calculated $\Delta q$ values are sufficient to adjust for the slow rotation of the Sun vector in inertial space. This allows the precession of the Zaxis to remain symmetric about the Sun vector. Thus, the combination of three rates-spin, precession, and Sun vector rotation-sweeps the instrument boresights over the entire sky in a period as short as six months.

Because of the large differences between the rates, this nominal science mode operates under the condition of being able to store momentum up to a specified magnitude but of an essentially arbitrary direction with respect to the body frame. With the loss of the use of a reaction wheel, however, the ability to store momentum in any arbitrary direction is lost. The two remaining wheels are limited to storing momentum in directions parallel to the plane spanned by their unit torque vectors.

The basic concept for an alternate control approach for a science mode using two wheels was presented by O'Donnell et al. ${ }^{2}$ The newly installed software would use thrusters to spin the spacecraft up to a momentum of approximately 20-25 newton-meterseconds (Nms) about the Sun vector. This momentum would create body rates of $2.3-2.9 \% \mathrm{sec}$, about the same total magnitude as the nominal science mode. With the momentum bias established, the backup algorithm would increase the Sun angle, $\theta$, to the desired value near $22.5^{\circ}$ and maintain that angle as well as possible. Thrusters would be used periodically to realign the momentum vector with the Sun vector so that the Sun angle would coincide with the nutation angle of the $Z$-axis about the momentum vector.

This approach is feasible because of the natural rigidbody dynamics determined by the mass properties of the MAP observatory. The current estimates for endof-life moments of inertia about the $\mathrm{X}$ and $\mathrm{Y}$-axes are nearly equal $\left(I_{x x}=572 \mathrm{~kg} \cdot \mathrm{m}^{2}\right.$ and $\left.I_{y y}=580 \mathrm{~kg} \cdot \mathrm{m}^{2}\right)$, and the moment of inertia about the $Z$-axis is smaller $\left(I_{z z}=496 \mathrm{~kg} \cdot \mathrm{m}^{2}\right)$, so that the body is prolate and essentially axisymmetric. The near-symmetry greatly reduces any effects a non-zero value of the $X Y$ product of inertia might have, so long as the $Z$-axis is in close alignment with the minor principal axis. As 
established by launch vehicle requirements, the geometrical $\mathrm{Z}$-axis is within $0.25^{\circ}$ of the minor principal axis, and misalignments of this magnitude have proved negligible in the development of this backup mode. Therefore, to simplify discussion, the axis frame denoted by $X, Y$, and $Z$ will refer henceforth to both the geometrical and principal axis frames simultaneously.

If we define a transverse moment of inertia, $I_{\mathrm{T}} \equiv \sqrt{I_{x x} \cdot I_{y y}}=576 \mathrm{~kg} \cdot \mathrm{m}^{2}$, then the ratio of the minoraxis $(Z)$ and transverse moments of inertia is $\sigma=0.86$ at end of life. At beginning of life, $\sigma=0.84$; because algorithm performance is insensitive to such small differences, a ratio of 0.86 is carried throughout. The ratio between the inertial nutation rate ( $\phi$-dot; analogous to precession of nominal mode) and body nutation rate ( $\psi$-dot; spin with respect to momentum vector) is:

For a nominal Sun angle of $\theta=22.5^{\circ}$, this ratio is approximately 0.15 ; for a system momentum of 20 Nms, the inertial nutation rate would be about $0.33 \mathrm{rpm}$, and the body nutation rate would be about $3 \mathrm{rph}$. The same ratio for the nominal scan mode was 60 ; an important effect of this difference is that the contingency scan pattern is less dense and interconnected. While undesirable, this data sparseness has been considered by MAP scientists not to be a great hindrance to contingency science data collection and processing.

Two-Wheel Science Requirements: Because of the difference between $I_{x x}$ and $I_{y y}$ the original requirement of $22.5^{\circ} \pm 0.25^{\circ}$ was no longer feasible using only two wheels, the requirements for this backup control law have been changed to match the priorities dictated by the science goals. Specifically, the heating of the instrument by sunlight is to be avoided above all other concerns, including the completeness of the current scan pattern. Also, to avoid heating of the instrument by diffracted sunlight, the Sun angle, $\theta$, would not be allowed to exceed $22.75^{\circ}$ during normal establishment of, and operations during, Two-Wheel Science Mode. If $22.75^{\circ}$ were exceeded, the algorithm would reduce $\theta$ as quickly as possible. To avoid sunlight falling directly on the instrument, $\theta$ will never be allowed to exceed $25^{\circ}$ under any circumstances. If an anomaly placed the Sun angle outside of $25^{\circ}$, the current Safehold mode would take over attitude control. Of lesser importance is a desire for the contingency mode to establish science operations within the nominal daily contact, so that system behavior may be observed immediately and corrections may be applied before returning to autonomous operations for the day.

Science Algorithm Design: The control algorithm design process was more expluratory, rather than strictly analytical. This approach was driven by factors such as nonlinear dynamics, underactuated control, a reduced set of state variables to be controlled (only $\theta$ need be controlled), and sparse literature from which to start. Due to limited software development resources, the algorithm was to fit into the same space in the software, with the same inputs and outputs, as the nominal science mode. The main effect of this restriction on the Two-Wheel science algorithm was that, since Sun angle was not already input into the nominal science mode controller, the backup science mode had to rely on the measurement of body and wheel rates to establish the angle between the $Z$-axis and the momentum vector. This reliance led to the creation of a new variable, the scan angle, which is the angle between the system momentum and the spacecraft $Z$-axis.

The general dynamics are governed by Euler's equation, which may be expanded in the following manner:

$$
\begin{aligned}
& \dot{\vec{H}}_{s \mathrm{w}}=-\vec{\omega}_{b} \times \vec{H}_{\mathrm{gpw}} \text { (body'frame) } \\
& \dot{\bar{H}}_{b}=-\dot{\bar{H}}_{R W_{A}}-\bar{\omega}_{b} \times \bar{H}_{b}-\bar{\omega}_{b} \times \bar{H}_{R W_{A}} \text {, } \\
& \text { with torque } \vec{T}_{\text {cymmand }}+\vec{T}_{\text {droo }}=-\dot{\vec{H}}_{R H^{\prime} /} \text {. }
\end{aligned}
$$

If the simplification is made that $I_{x x}=I_{y y}$, then the control-free motion may be parameterized using the scan angle, $\theta^{*}$, and the azimuthal angle for the momentum in the body frame, $\psi^{*}$ :

$$
\begin{aligned}
& H_{x}=H \sin \theta^{*} \cos \psi^{*} \\
& H_{y}=H \sin \theta^{*} \sin \psi^{\circ} \\
& H_{z}=H \cos \theta^{\circ} \\
& \psi^{*}=\dot{\psi}^{*} t+\psi_{t=0}^{*} .
\end{aligned}
$$

Here, $\mathrm{H}$ is the magnitude of the system momentum and remains constant as the system momentum vector sweeps a cone-like surface in the rotating body frame; if $\theta^{*}$ is constant, then the surface is truly a cone. The angles $\theta^{*}$ and $\psi^{*}$ are analogous to $\theta$, the Sun angle, and $\psi$, the angle between the $X$-axis and the XY-projection of the Sun vector. When the Z-axis is aligned with the Sun vector, $\theta=\theta^{*}$. Therefore, it would be sufficient to control the scan angle as though it were the Sun angle as long as the Sun vector were kept close to the momentum vector; this function would be performed by the Momentum Adjust algorithm. 
Note that, since the motion of the body momentum vector is nearly symmetric about the $Z$-axis, and the wheels are also symmetric about the $Z$-axis, a geometrical simplification may be used to make one general control law for any wheel failure. The failed wheel must be identified (RWA1, 2, or 3) and its identity entered into the software by means of a table value (the algorithm relies on the failed wheel being immobilized). Then, the algorithm may redefine the body frame such that the failed wheel is located on the new positive $X$-axis, which may be called the $X^{*}$. axis. The $Z$-axis is identical with the $Z^{\circ}$-axis, and the $\mathrm{Y}$-axis is rotated into the $\mathrm{Y}^{*}$-axis accordingly. The operable wheels are then situated symmetrically about the $Z X^{*}$-axis. The subscript $\alpha$ will be used here to denote the $-Y^{*}$-wheel, and $\beta$ will refer to the $+Y^{*}$-wheel. Variations in behavior for the failure of each wheel were not large, so small changes the algorithm parameters for each case have been sufficient to achieve good all-around performance.

The various attempts to find a backup algorithm resulted in several simple one-wheel and two-wheel algorithms based on error functions defined as:

$$
\begin{aligned}
& \text { Transverse error: } e_{r} \equiv H_{b} \sin \theta^{*}-H_{h \text {.desired }} \sin \theta_{\text {devired }}^{*}, \\
& Z \text {-axis error: } \quad e_{Z} \equiv H_{b} \cos \theta^{*}-H_{h, \text { deired }} \cos \theta_{\text {devired }}^{*} .
\end{aligned}
$$

The control algorithms were composed of three parts: an initial kick to escape limit cycles about the Sun vector, an error-reducing function of the measured body momentum, and an anti-runaway restriction on the speed of the wheels. The expressions used for each of these parts is:

Anti-Runaway: $\omega_{i} \geq \omega_{\mathrm{lim}}, i \in\{\alpha, \beta\} \Rightarrow T_{i}=-K_{A R} \omega_{i}$.

Sun Escape: $\quad \theta<10^{\circ} \Rightarrow T_{\alpha}=T_{\beta}=T_{\text {escape }}$.

Error-Reduction: $T_{\alpha}=k_{z \alpha} e_{Z}+k_{7 \alpha} e_{T} \cos \left(\psi^{*}+\varphi_{\alpha}^{*}\right)$ $T_{\beta}=k_{Z \beta} e_{Z}+k_{T \beta} e_{T} \cos \left(\psi^{*}+\varphi_{\beta}^{*}\right)$.

The control gains and other parameters selected were:

$$
\begin{gathered}
k_{Z \alpha}=0.007 \mathrm{Nm} / \mathrm{Nms}, \\
k_{T \beta}=0.010 \mathrm{Nm} / \mathrm{Nms}, \\
k_{Z \beta}=k_{T \alpha}=0 \mathrm{Nm} / \mathrm{Nms}, \\
\varphi_{t z}=0^{\circ}, \varphi_{\beta}=30^{\circ}, \omega_{i \mathrm{in}}=50 \mathrm{rpm} .
\end{gathered}
$$

This control law was tested using high-fidelity Monte Carlo simulations to establish the stability of the controller.
Two-Wheel Mode Performance: The figures presented at the end of this section show simulated operation of the science mode algorithm. Figures 2 and 3 show a case in which RWAl has failed, the spacecraft has been recovered by Safehold/CSS, and the thrusters have established a momentum bias of $20 \mathrm{Nms}$ parallel to the Sun vector. The algorithm shows good stability and performance for initial conditions over the full range of the cone defined by $\theta^{*} \leq 25^{\circ}$; in the illustrated case, the scan angle starts at $10^{\circ}$. When the natural motion reduces the scan angle below the $10^{\circ}$ Sun Escape limit, the Sun Escape torques quickly bring the wheels up to their speed limit. At the speed limit, the Anti-Runaway portion of the algorithm strongly counteracts any increase in wheel speed, and a switching torque pattern begins. The essentially constant speed of the wheels acts to drive the $Z$-axis away from the Sun vector.

Starting from Sun angles less than the desired value, the algorithm establishes the desired motion in approximately 6-10 minutes, as is demonstrated in Figure 2. Since the nominal ground contact during science operations is supposed to last 37 minutes, this time is well within the goal of establishing science operations within a single contact.

Though analytical modeling of fuel slosh for MAP indicates that the energy dissipation caused by slosh during this natural motion mode would be very small, it is possible that those models are in error. Because the Z-axis is the minor principal axis, such energy dissipation would cause the Sun angle to increase. No simulation of fuel slosh dissipation was conducted; testing the ability of the controller to recover from large Sun angles was deemed adequate. Simulations were run for cases in which the Sun angle exceeded the allowable range for science and was close to the dangerous range $\left(\theta>25^{\circ}\right)$ where direct Sunlight would heat the instrument. Ideally, the Sun angle would never reach this value; nevertheless, the control algorithm must be and is able to recover and to stabilize the Sun angle quickly about the desired value. During operations at high Sun angle, the error reduction portion of the algorithm is generally the only active portion, as the wheel speeds do not exceed the Anti-Runaway limits. 


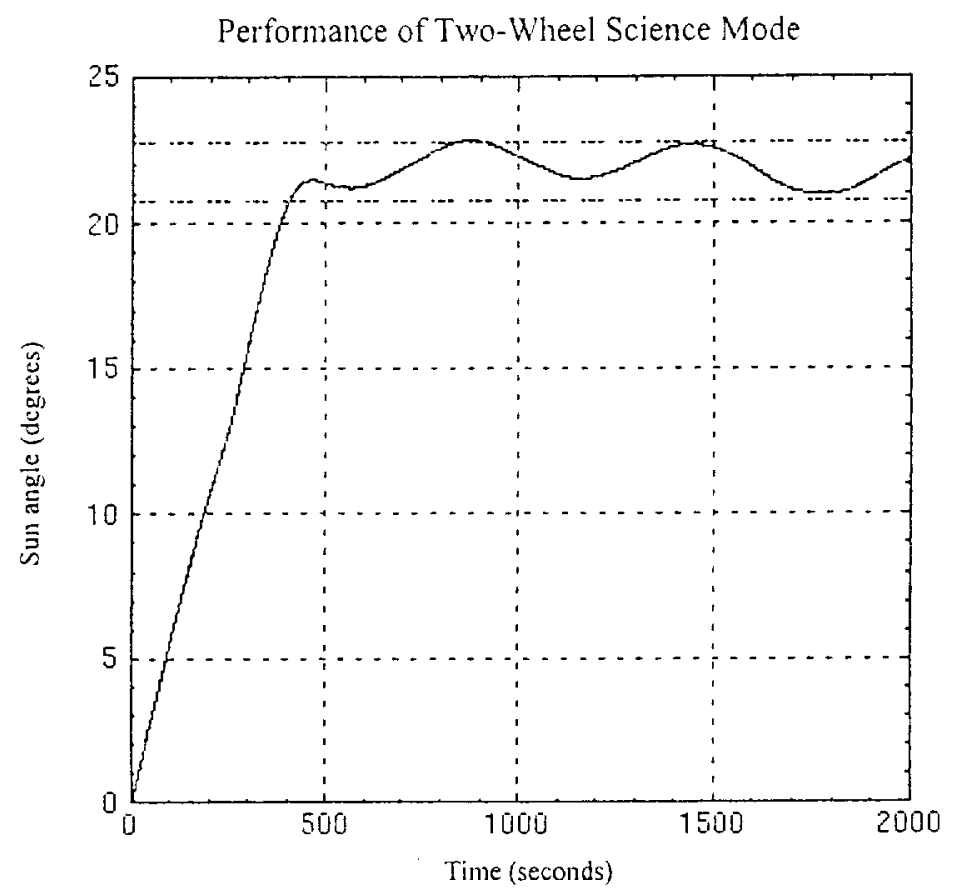

Figure 2: The jagged torque profile shows typical commands during Sun Escape, a portion of the Two-Wheel Science Mode algorithm which is used to avoid limit cycles at low Sun angles. After error reduction, the mode is established, and torque commands become unnecessary unless the Sun angle leaves the operating range.

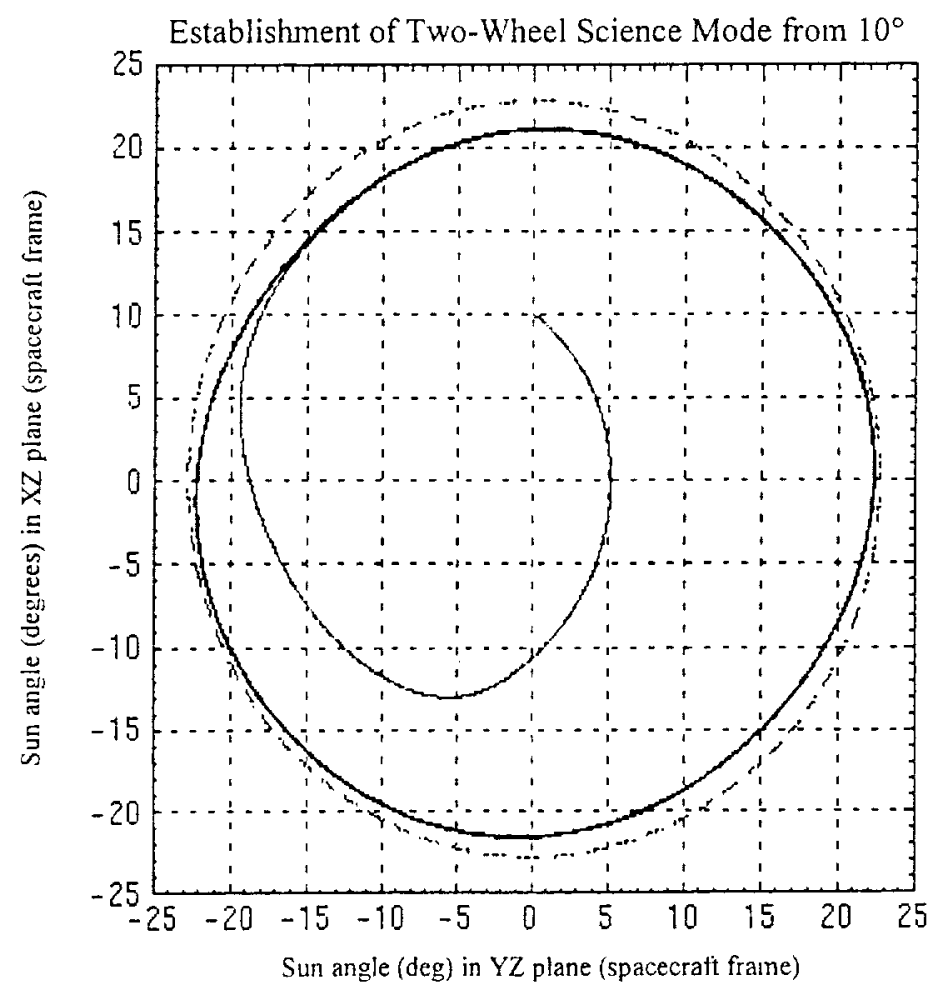

Figure 3: Establishment of mode showing the trajectory of the Z-axis direction. The Sun is located at the origin; the dashed circle shows the $22.75^{\circ}$ performance limit. 


\section{Momentum Adjust Algorithm}

The momentum bias vector used for the Two-Wheel Science Mode can be placed about the Sun vector initially. However, because of the orbital motion of the spacecraft about the Sun, the angle between the momentum vector and the Sun vector changes by approximately $1 \%$ day. To keep the science instrument safely behind the Sun shield and to sweep the scan pattern around the sky, the momentum vector needs to be adjusted to stay nearly aligned with the Sun vector. It is least disruptive to science data collection to adjust the momentum during the daily contact with the spacecraft.

To simplify operations and minimize the amount of fuel used, the Momentum Adjust algorithm was designed to be used while the Observatory is spinning in the science mode. Once enabled, the algorithm would wait for the selected spacecraft thruster to be oriented such that a short thruster firing would move the momentum vector from the measured inertial location to the desired alignment with the Sun vector.

System Momentum Measurement: The Momentum Adjust algorithm depends on an accurate measurement of the spacecraft system momentum. Because the wheel tachometers have significant measurement and quantization noise, however, it is difficult to get a good measurement. Fortunately, there is a way to alleviate this problem. Nominally, the two-wheel science mode keeps the functioning reaction wheels at speeds less than about $50 \mathrm{rpm}$. Because the mode uses the spacecraft natural motion to establish the dual spin and because of low levels of energy dissipation from fuel slosh and other effects, it is permissible to turn off wheel control for limited periods of time. Since the maximum wheel speeds during science mode are limited to $\sim 50 \mathrm{rpm}$, by turning off wheel control and zeroing torque commands to the wheels, they will run down to zero wheel speed fairly quickly. Once the wheels have stopped moving, the spacecraft system momentum can be measured very accurately using only the estimated body rates provided by the onboard Kalman filter.

Thruster Firing Direction and Timing: There are three vectors that define the desired thruster firing direction for adjusting the momentum bias. The only measured vector of the three describes the direction in inertial space of the momentum bias itself. To move this vector, its current orientation must be known along with the other two vectors: the desired orientation and the thrust direction of the appropriate thruster must be known. Whereas the momentum vector in inertial space is important, the reference frame used for the three direction vectors is not, as long as only one frame is used. Because all sensor measurements are defined in the body frame, that frame is the easiest one in which to perform the calculations.

Figure 4 shows a schematic of the method used to determine the thruster firing direction and timing. Because the thruster directions are fixed and the momentum adjustments will be done while the spacecraft is spinning, the determination of direction ends up being a matter of deciding when to command a thruster firing. The decision-making algorithm consists of waiting for the wheels to run down, detecting the proper time at which to fire the thruster, and firing for up to one second (one control cycle). The method used to calculate the time and duration of the thruster firing are detailed below:

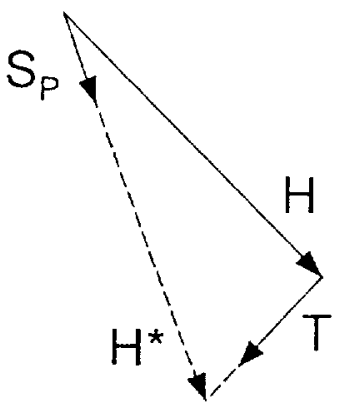

Figure 4: Momentum Adjust Thruster Direction

Nominally, the goal of the Momentum Adjust algorithm is to align the momentum vector with the Sun vector. However, given that these adjustments will be done approximately once a day, it makes sense to bias the desired vector on the other side of the Sun vector. In the geocentric inertial reference frame used by MAP, the Sun vector moves approximately $1 \%$ day. To center the actual Sun vector during the time between momentum adjustments, the target vector can be biased off of the Sun vector using this rate; the resulting target unit vector is denoted $S_{P}$, and the resulting momentum bias vector is denoted $\mathrm{H}^{*}$. T is defined as the torque provided by a chosen thruster; it is also, therefore, the momentum change provided by a one-second firing of that thruster.

In Figure 4 , the $H$ vector and unit vector $S_{p}$ define the actual and desired directions of the momentum bias vector, and these two vectors form a plane. As the spacecraft rotates in the Two-Wheel Science 
Mode, any selected thruster torque vector will become parallel to the plane defined by $H$ and $S_{p}$, meaning that a thruster firing of the appropriate duration at that time will move the $H$ vector to align with $S_{p}$. This happens when the thruster torque vector, $T$, is parallel to the plane; i.e. when the quantity $\left(\mathrm{H} \times \mathrm{S}_{\mathrm{P}}\right) \cdot T$ is equal to zero.

Because the natural spacecraft motion in Two-Wheel Science mode is a dual spin greater than $2 \%$ second and because the control cycle is 1 second, it is not possible to calculate the correct time exactly. Therefore, the Momentum Adjust algorithm looks for a zero-crossing of the product $H \times S_{p} \bullet T$. When such a zero-crossing is detected, a firing opportunity for momentum adjustment is present. It is then determined whether the appropriate thruster is in the desired orientation for the Momentum Adjust or $180^{\circ}$ out of phase.

Duration of Thruster Firing: Once the timing of the thruster firing has been determined, the duration of the firing can be calculated. If the thruster is in the correct orientation, the calculated thruster firing will be positive; otherwise, it will be negative. Looking again at Figure 4, one can see that

$$
H+\tau T=H^{*}=\sigma S_{p},
$$

where $\tau$ is the magnitude of the momentum change vector to be added by the desired thruster firing. Of the four MAP thrusters used for momentum adjust, two are oriented to provide primarily $\mathrm{X}$-axis torques and the other two provide $\mathrm{Y}$-axis torques. For thrusters 1 and 2 , the $X$-axis thrusters, expressing this equation in the $X Z$-plane yields:

$$
\begin{aligned}
& H_{X}+\tau T_{X}=\sigma S_{P X} \\
& H_{Z}+\tau T_{Z}=\sigma S_{P Z}
\end{aligned}
$$

Solving each equation for $\sigma$ gives:

$$
\sigma=\left(H_{x}+\tau T_{x}\right) / S_{P X}=\left(H_{z}+\tau T_{z}\right) / S_{P z}
$$

This equality then can be solved for $\tau$ :

$$
\tau_{1.2}=-\left(H_{X} S_{p Z}-H_{z} S_{p X}\right) /\left(T_{X} S_{P Z}-T_{z} S_{p X}\right)
$$

For the $Y$-axis thrusters, thrusters 3 and 4 , the analogous expression for calculating $\tau$ is:

$$
\tau_{3.4}=-\left(H_{Y} S_{P Z}-H_{Z} S_{P Y}\right) /\left(T_{Y} S_{P Z}-T_{Z} S_{P Y}\right)
$$

In a Momentum Adjust maneuver, the MAP thrusters will be used in a one-shot fashion, meaning that the firing will be limited to no more than one second. Because $T$ represents a thruster torque direction and the magnitude of momentum change provided by a one-second firing of that thruster, the calculated value of $\tau$ will be between 0 and 1 for a one-shot thruster command. Because thruster commands on MAP are expressed as a number of 40 -millisecond pulses, the Momentum Adjust command in pulses is found by calculating $25 \tau$ and rounding it to the nearest integer.

Algorithm Refinements: By following the two steps above-that is, by calculating the timing and duration of thruster firing for Momentum Adjust-the algorithm finds a valid firing opportunity. The resulting momentum bias vector $\mathrm{H}^{*}$ will not necessarily be the same magnitude as the original vector $\mathrm{H}$. Changing the direction of the momentum vector keeps the science mode spin oriented correctly with respect to the Sun, but changing the magnitude of the vector will increase or decrease the rate of the spin. It is desirable from a science standpoint to keep the spin rate relatively constant; small variations are acceptable, but as they get larger it negatively impacts the processing of the science data. To minimize the change to the magnitude of the system momentum vector, a refinement of the algorithm was sought.

Firing Opportunity Selection: The refinement should select among thruster firing opportunities to either minimize the change in spacecraft rates and/or determine that the system momentum magnitude should increase or decrease from its initial value. It might be possible to keep the magnitude nearly constant by firing more than one thruster to do a Momentum Adjust, but this would make the algorithm much more complicated. To keep in line with the design philosophy stated near the beginning of this paper, a less complex solution was found.

Considering first the $Y$-axis thrusters 3 and 4 , because they are oriented to produce almost exclusively $\mathrm{Y}$-axis torques, the change in the magnitude of the momentum bias caused by firing one of these thrusters depends on where in the body the momentum bias is located. Therefore, for these thrusters, a firing opportunity is selected by first detecting a zero-crossing of $\left(H \times S_{p}\right) \bullet T$. Then, for each opportunity identified, the $Y$-axis component of the momentum bias, $H_{Y}$, is saved to look for a zerocrossing of $\mathrm{H}_{Y}$ from one firing opportunity to the next; the zero-crossing indicates that the quantity is relatively small. By further looking for a negative-topositive zero-crossing $\left(\mathrm{H}_{Y} \mathrm{H}_{Y O L D}<0\right.$ and $\left.\mathrm{H}_{Y}>0\right)$, the thruster selection of 3 or 4 will produce an increase or decrease in the magnitude.

Because thrusters 1 and 2 are canted $10^{\circ}$ from the $\mathrm{YZ}$-plane and so do not produce a pure $\mathrm{X}$-axis torque, the derivation of how to select a firing opportunity is not as straightforward. The method 
chosen was somewhat empirically determined. As it turns out, the same method can be used as with thrusters 3 and 4 , except that the two conditions needed are $H_{\gamma} H_{\text {YoL }}<0$ and $H_{x}>0$. In testing, the $\mathrm{H}_{Y}$ zero-crossing was more effective than the $\mathrm{H}_{X}$ zero-crossing, so the only difference between the two sets of the thrusters is that the condition $\mathrm{H}_{x}>0$ is used in the calculation for thrusters 1 and 2 , and $H_{Y}>0$ is used in the calculation for thrusters 3 and 4 . The calculations can be combined in the algorithm implementation by creating a quantity $\mathrm{H}_{\mathrm{CHECK}}$ and setting it equal to $\mathrm{H}_{X}$ when the selected thruster is 1 or 2 and $H_{Y}$ for 3 or 4.

With the resulting algorithm, thrusters 1 and 3 increase the magnitude of the momentum bias and thruster 4 decreases the magnitude of the momentum bias. Also, though thruster 2 may occasionally increase the momentum by a small amount, it usually decreases the momentum magnitude.

Once it has been decided whether to bias towards an increase or a decrease in momentum, the thruster selection may be made based on orbit considerations. Thrusters 1 and 2 push the spacecraft away from the Sun and thrusters 3 and 4 push it towards the Sun. Since nominal stationkeeping maneuvers near $\mathrm{L}_{2}$ require sunward or anti-sunward thrust, it is hoped that the daily momentum adjustments could be selected to eliminate the need for additional stationkeeping operations. This would save fuel and extend the mission by eliminating the need to remove the momentum bias for each stationkeeping burn and then spin the spacecraft up again after the burn.

The advantage of using the refinement described above is that it minimizes the momentum bias magnitude change and allows the direction of the magnitude change to be selected. The disadvantage is that it might take longer for the Momentum Adjust algorithm to execute the thruster firing.

Fast Fire Flag: Because of the precession and spin rates of the two-wheel spin, a firing opportunity occurs approximately every two minutes. Using the refined algorithm, a firing opportunity will be selected within approximately twenty minutes of the Momentum Adjust being commanded. A fast fire flag has been included in the algorithm to overcome this disadvantage; if a faster Momentum Adjust firing is operationally desirable, the flag can be set and the first firing opportunity will be used.

Momentum and Target Vector Propagation: Because the algorithm can only fire the thrusters up to one second, it would be beneficial to be able to fire in the proper cycle, rather than one cycle late. The dual spin motion used in the Two-Wheel Science Mode is much less uniform than that of the nominal science mode. Nevertheless, over periods of one or two seconds, the body rates are relatively constant. It is possible to use the estimated gyro rates to propagate the momentum vector measurement and target vector calculation forward a short time-nominally between 0 and 1 second. This allow's the thruster timing to be determined before the optimal firing time has passed, and should make the resultant Momentum Adjust more accurate. The vectors are propagated forward using an approximation of the transport theorem given by the equations:

$$
\begin{aligned}
& H_{\text {PROP }}=H-\alpha_{H}(\omega \times H) \\
& T_{\text {PROP }}=T-\alpha_{T}(\omega \times T)
\end{aligned}
$$

The parameters $\alpha_{H}$ and $\alpha_{T}$ are in units of time and used as propagation factors to prescribe how far ahead to propagate the two vectors. Nominally, these factors would be selected for propagation of $0-1$ seconds because the approximation is valid only for small values of $\alpha$. The software implementation of the algorithm allows for the two factors to be different, but they most likely will be set equal to one another.

Momentum Adjust Performance: Testing of the Momentum Adjust algorithm has been performed using the same high-fidelity dynamic simulation environment as was used for the nominal ACS modes. The thruster used for the maneuver was varied among the four allowed, and the fast fire flag status was tested to check for improvements in momentum magnitude control. The propagation factors were varied as well, but they were always kept the same as each other; i.e. $\alpha_{H}=\alpha_{T}$. The testing of these parameters was part of the acceptance testing of the second-phase software patch; testing on that patch was nearly completed at the writing of this paper. Once the patch is satisfactorily tested, it will be ready for upload to the spacecraft at any time in the future of the mission that the MAP operations team decides a wheel must be deactivated.

Figure 5 shows the accuracy of Momentum Adjusts simulated for firings of thruster 1 . The circles mark the targeted orientations of the momentum biases, and the triangles indicate the actual orientations after the firings; there were multiple tests of each commanded bias. The figure shows all final orjentations to lie within $0.4^{\circ}$ of the targeted orientation; over all tests conducted for all four thrusters, the standard deviation of the error angle between desired and actual bias orientations was about $0.1^{\circ}$. In these tests, the system momentum magnitude increased or decreased as described above. 


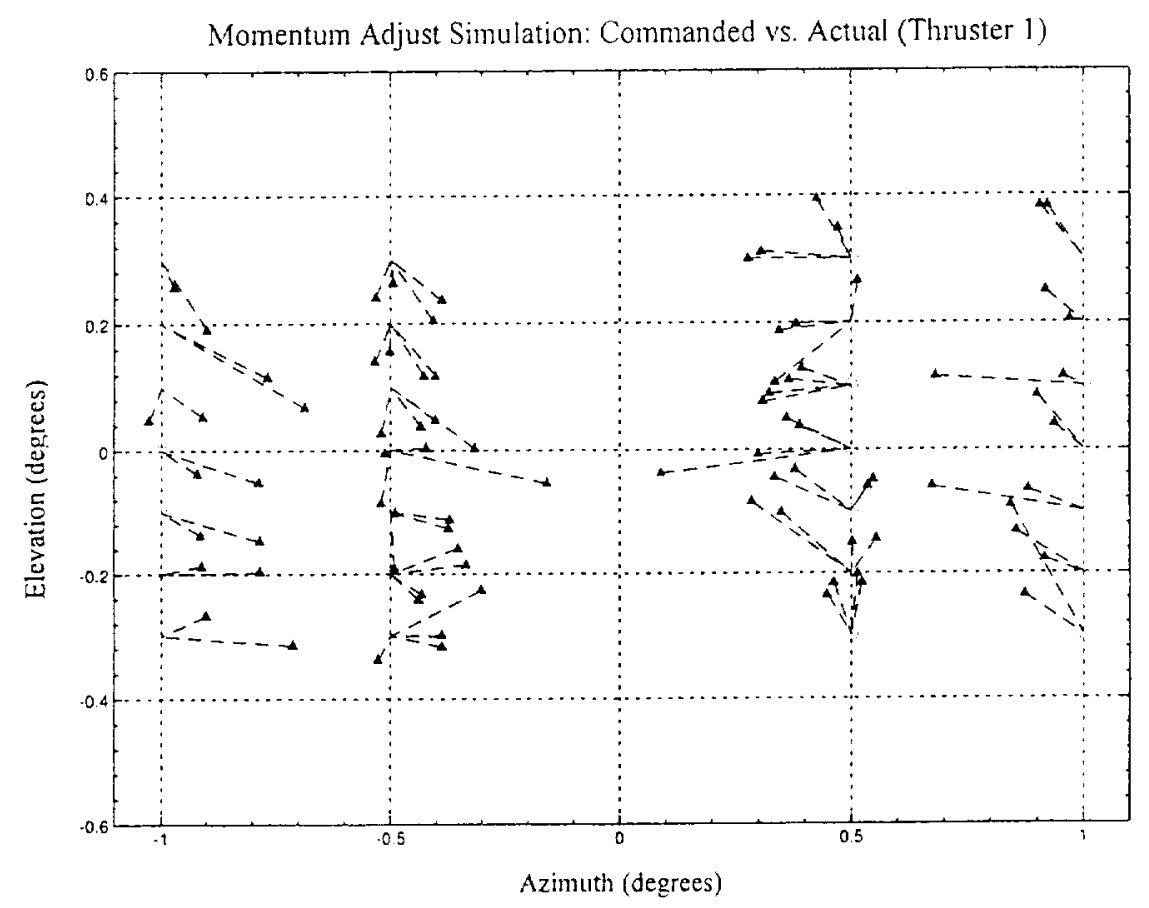

Figure 5: Results of simulations of the Momentum Adjust algorithm, which uses body rate information to calculate the timing and duration of a single-control-cycle, single-thruster burn. In the figure, circles mark the commanded orientation of the spacecraft momentum bias, and the triangles connected to the circles by dashed lines indicate the orientation after the simulated thruster firings. Orientations are given in angles for azimuth (X-component) and elevation ( $\mathrm{Y}$-component).

\section{Summary}

Due to cost and mass budget considerations, the MAP Observatory has no redundant reaction wheel to back up the three nominally operational wheels. In the event of a wheel failure, it is possible to perform all of the necessary attitude control using thrusters and the two remaining wheels. This paper has briefly described a Thruster-Based Inertial Mode, which would have used thrusters to slew for critical burns; the necessary software changes were available at launch for immediate upload had a wheel failed before the spacecraft reached $L_{2}$.

A Two-Wheel Science Mode has been detailed in which a thruster-generated momentum bias is used to sweep the instrument boresights across the sky in a manner similar to the nominal science mode. However, this natural-motion science mode results in a decrease in data density over the sky.

The momentum bias must point at the Sun as much as is feasible; so, it must be adjusted by the thrusters often for the science mode to operate safely and effectively. A new Momentum Adjust algorithm was designed to control the thruster during these daily operations.

Because of the intractability of the attitude control problem, the science mode algorithm has been designed to allow for the adjustment of several parameters, including angle error gains, and other parameters used to adjust various failsafe modes built into the algorithm. All of these parameters may be adjusted in a flight software table that is part of the existing architecture. This design philosophy allows a measure of security against unpredictable dynamical effects.

\section{References}

1. S. F. Andrews, C. E. Campbell, A. J. EricssonJackson, F. L. Markley, J. R. O'Donnell, "MAP attitude control system design and analysis," Flight Mechanics and Estimation Theory Symposium, NASA Goddard Space Flight Center, Greenbelt, MD, May 1997.

2. J. R. O'Donnell, S. F. Andrews, A. J. EricssonJackson, T. W. Flatley, D. K. Ward, P. M. Bay, "Backup attitude control algorithms for the MAP spacecraft," Flight Mechanics Symposium, Greenbelt, MD, 1999

3. S. R. Starin and J. R. O'Donnell, "A TwoWheel Observing Mode for the MAP Spacecraft," Flight Mechanics Symposium, Greenbelt, MD, 2001. 